\title{
Cuerpo extraño gastrointestinal en niños
}

\author{
M. P. Muñoz $・$ R. Maluje $・$ F. Saitua
}

\section{ABSTRACT \\ Gastrointestinal foreign body in children}

Gastrointestinal foreign bodies (FB) are frequent complaints in the emergency departments, with esophageal foreign bodies as the most frequent cases. Symptoms are varied and depend on the location of the FB, with several techniques available for removal. Objective: To describe the FB removed by endoscopy in children as well as the consulting population characteristics. Patients and Method: Patient chart reviews of those who, during the last six years, underwent gastrointestinal endoscopy were performed. Descriptive statistics were used to analyze the data. Results: 51 patients, 28 males and 23 females, with average age of four years old, underwent endoscopy due to FB intake. The most common symptoms were drooling (45\%) and dysphagia (35\%); the FBs were extracted mostly under general anesthesia. Coins were the most common foreign bodies found $(57 \%)$ and they were mainly located in the esophagus $(91 \%)$. All bodies were extracted by direct endoscopy or endoscopically assisted extraction; lesions that corresponded to erosions were found in 19 (37\%) patients and ulcers in $4(8 \%)$. There were no complications. Conclusions: Endoscopic removal of gastrointestinal FBs is a safe procedure. Given the risk of serious injury, especially in foreign bodies in the esophagus, performing endoscopy within a reasonable time must always be considered. Management protocols are necessary to unify the standards of those working in emergency rooms, having in mind that the most important strategy is prevention.

(Key words: Endoscopy, foreign body, pediatrics, gastrointestinal).

Rev Chil Pediatr 2014; 85 (6): 682-689

\section{RESUMEN}

Los cuerpos extraños (CE) gastrointestinales son motivo de consulta frecuente en los servicios de urgencia, siendo los esofágicos los de mayor riesgo Los síntomas de presentación son variados y dependen de la ubicación del CE, existiendo diversas técnicas para su extracción. Objetivo: Describir las características de la población consultante y de los CE retirados por endoscopia en niños. Pacientes y Método: Se realizó una

Recibido el 05 de noviembre de 2013. Última versión aceptada el 14 de noviembre de 2014.

María Paz Muñoz F. ( $ه$ ), Rodrigo Maluje J., Francisco Saitua D.

Unidad Gastroenterología y Nutrición Infantil, Hospital Dr. Luis Calvo Mackenna.

E-mail:pazmunioz@gmail.com

María Paz Muñoz F., Francisco Saitua D.

Departamento Pediatría y Cirugía Infantil, Facultad de Medicina, Universidad de Chile.

Rodrigo Maluje J.

Unidad de Cirugía Infantil, Hospital Dr. Gustavo Fricke, Valparaíso.

Francisco Saitua D.

Servicio Cirugía Infantil, Hospital Dr. Luis Calvo Mackenna, Santiago, Chile. 
revisión de los últimos 6 años, de las fichas de los pacientes a los que se le indicó una endoscopía digestiva por CE gastrointestinal. Se utilizó estadística descriptiva para el análisis de los datos. Resultados: 51 pacientes, 28 hombres y 23 mujeres, con un promedio de edad de 4 años, fueron sometidos a endoscopía digestiva por ingesta de CE. Los síntomas de presentación más frecuentes fueron la sialorrea $(45 \%)$ y la disfagia $(35 \%)$, siendo extraídos los CE en su mayoría con anestesia general. Los CE más frecuentemente fueron monedas (57\%) y se ubicaron principalmente en esófago (91\%). Todos se extrajeron mediante endoscopía directa o con asistencia endoscópica y las lesiones encontradas fueron erosiones en 19 (37\%) pacientes y úlceras en 4 (8\%). No hubo complicaciones. Conclusiones: La extracción endoscópica de CE gastrointestinales es un procedimiento seguro. Dado el riesgo de lesiones graves, especialmente en los CE de ubicación esofágica, debe considerarse siempre la realización de la endoscopía dentro de un plazo prudente. Los protocolos de manejo son necesarios para unificar los criterios de las personas que trabajan en los servicios de urgencia teniendo presente que la estrategia más importante es la prevención.

(Palabras clave: Endoscopia, cuerpo extraño, pediatría, gastrointestinal).

Rev Chil Pediatr 2014; 85 (6): 682-689

\section{Introducción}

La ingestión de cuerpos extraños (CE) es un motivo de consulta frecuente en los Servicios de Urgencia Pediátricos. Se describe que en la edad pediátrica se presenta el $80 \%$ de los $\mathrm{CE}$, observándose principalmente entre los 6 meses y los 3 años de edad ${ }^{1}$ y constituye la segunda causa de endoscopia digestiva urgente en pediatría ${ }^{2}$. En niños habitualmente la ingesta es accidental, siendo especialmente frecuente en aquellos que presentan factores predisponentes como trastornos neurológicos, psiquiátricos, enfermedades neuromusculares, estenosis esofágica previa, esofagitis eosinofilica o enfermedad péptica ${ }^{3-5}$.

En Estados Unidos se reporta una incidencia anual de 100.000 casos en este grupo etario $^{1,6}$. La mortalidad asociada a la ingestión de CE es muy baja, siendo $1 / 2.206$ casos anuales en población pediátrica, según grandes estudios multi céntricos ${ }^{1,4,7}$.

Hay una amplia variedad de CE descritos en las diferentes publicaciones, destacando, en primer lugar las monedas, seguido de partes de juguetes, pilas, objetos punzantes, y espinas de pescado o huesos de pollo en menor frecuencia, cercanos al 5-30\% $\%^{3,6,8-10}$. En nuestro país también existen publicaciones que señalan que las monedas son la primera frecuencia entre los diferentes tipos de $\mathrm{CE}^{11}$; sin embargo, lo anterior depende el país de residencia, en Asia por ejemplo, dado el alto consumo de pesca- do, la ingesta de espinas de pescado ocupa el primer lugar y las monedas el segundo lu$\operatorname{gar}^{2,6}$. En adultos la impactación por alimentos principalmente carne puede ser frecuente, sin embargo en niños la retención de alimentos en esófago es infrecuente y puede ser la primera manifestación de una enfermedad del esófago como las esofagitis eosinofilica o patología esofágica subyacente $\mathrm{e}^{3,6,8,12}$.

Sólo el 10-20\% de los niños requiere de intervención (extracción endoscópica) que generalmente es exitosa ya que la mayoría de los $\mathrm{CE}$ gastrointestinales corresponde a objetos romos y pequeños ${ }^{13}$. Sólo se describe un $1 \%$ que pudiese requerir cirugía ${ }^{2}$. El diagnóstico es tardío en un porcentaje pequeño de pacientes y pueden suceder complicaciones en un $1-3 \%^{1,12}$.

En el esófago, los CE se localizan principalmente en 3 zonas (músculo cricofaríngeo, cayado aórtico y esfínter esofágico inferior) y su sintomatología varía desde ser asintomáticos o sensación de CE, náuseas, vómitos, tos, dificultad respiratoria, sialorrea y sangrado. Los CE gástricos e intestinales en general son menos sintomáticos, incluso puede ser un hallazgo radiológico en un paciente asintomático, o manifestarse por una complicación en la cual no se sospechaba la ingesta de CE. Es por esto que los síntomas no deben orientar la conducta terapéutica ${ }^{14}$.

El objetivo de este estudio es describir las características de los pacientes que consultan en el Hospital Luis Calvo Mackenna (HLCM) 
por sospecha de ingesta de cuerpo extraño que requieren extracción endoscópica, la frecuencia de cada uno de los diferentes tipos de cuerpo extraño, los hallazgos endoscópicos, método de extracción y frecuencia de complicaciones asociadas.

\section{Pacientes y Método}

Se realizó una revisión de todas las fichas clínicas de pacientes sometidos a endoscopía digestiva alta (EDA) que consultaron en Servicio de Urgencias HLCM por sospecha de ingesta de CE y en cuyo registro digital se incluyó las palabras "cuerpo extraño", entre junio de 2007 y junio de 2013. Para el análisis de los datos se incluyó sólo aquellos pacientes con el antecedente o la sospecha de ingesta, excluyendo aquellos en que el cuerpo extraño fue un hallazgo de la EDA, indicada por otra causa.

Los datos registrados fueron edad, sexo, síntomas de presentación, tiempo transcurrido entre la ingesta del CE y el procedimiento endoscópico, ubicación, tipo $\mathrm{CE}$, número $\mathrm{CE}$, características radiológicas, forma de extracción, presencia de lesiones secundarias post endoscopia y presencia de complicaciones asociadas al procedimiento.

Este protocolo fue revisado y aprobado por comité de ética del Servicio de Salud Metropolitano Oriente, Santiago, Chile.

Se utilizó estadística descriptiva (medias, frecuencia y porcentajes) para resumir los hallazgos, utilizando el software Statview.

\section{Resultados}

Se revisaron 54 fichas y se excluyeron 3 de ellas por presentar registros incompletos. De los 51 pacientes incluidos $55 \%$ eran de sexo masculino y $45 \%$ femenino.

La mayoría de los pacientes eran sanos, sólo 3 pacientes $(6 \%)$ presentaban antecedentes médico quirúrgicos relevantes (un paciente con Síndrome de Down y retraso del desarrollo psicomotor, un niño con retraso psicomotor de origen desconocido y atresia esofágica operada y otro niño con estenosis esofágica secundaria a enfermedad por reflujo gastroesofágico.

La edad promedio de los pacientes fue de 4 años 2 meses, siendo el menor de ellos un lactante de 5 meses y el mayor un adolescente de 16 años de edad.

El $94 \%$ de los pacientes tenía el antecedente de ingesta, $45 \%$ se presentaron con sialorrea, $35 \%$ tenía disfagia u odinofagia, un porcentaje menor $(12 \%)$ dificultad respiratoria y/o tos, $14 \%$ vómitos, $4 \%$ sensación de cuerpo extraño, $4 \%$ halitosis y un paciente $(2 \%)$ se presentó con fiebre. Como algunos de los niños presentaban más de un síntoma, se consideró cada síntoma en forma individual para el cálculo de los porcentajes (figura 1).

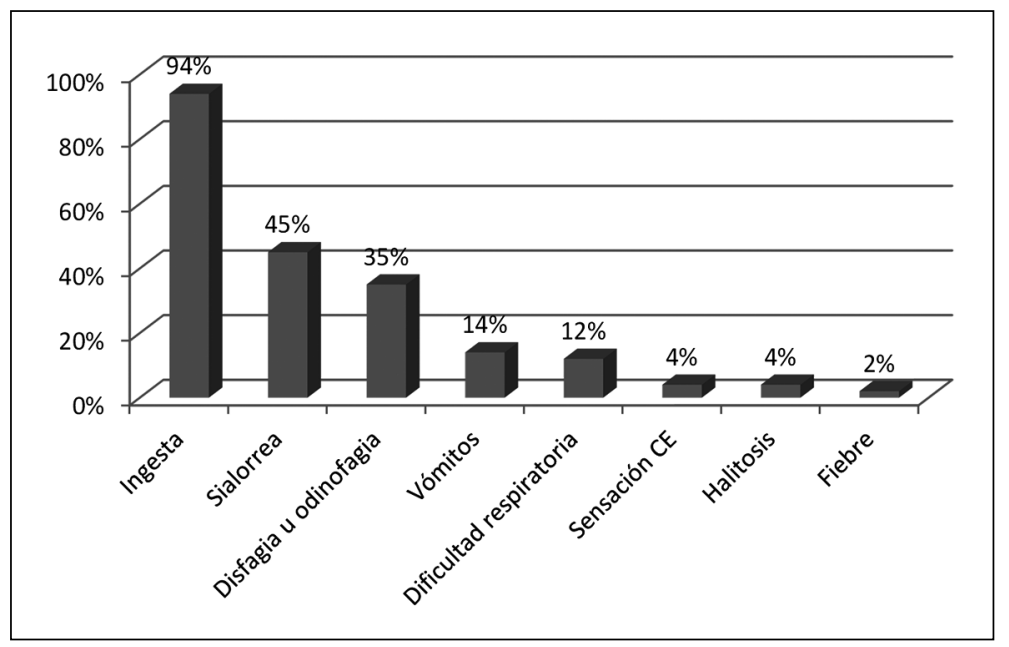

Figura 1. Distribución de los síntomas de presentación de 51 pacientes con cuerpo extraño digestivo. 
A todos los pacientes se les realizó radiografía cervical, torácica y abdominal en posición lateral y frontal a su ingreso. Según la radiografía inicial, un 25\% eran radio lúcidos y $75 \%$ radiópacos. En cuanto a la ubicación del $\mathrm{CE}$ en el primer estudio radiológico, $88 \%$ se encontraban en esófago, $10 \%$ en estómago y $2 \%$ en duodeno.

Del total de EDAs, no se encontró CE en 5 (10\%) pacientes por migración del mismo, 3 de los cuales tenían diagnóstico de CE esofágico y 2 de CE gástrico radiológico (radiografía simple) a su ingreso. De los 46 pacientes en que sí se encontró CE durante la EDA, la gran mayoría $63 \%$ se encontraron en el tercio superior del esófago, $24 \%$ tercio medio y $4 \%$ tercio inferior. Sólo el 9\% de los CE se encontraron más distales; 3 en estómago y 1 paciente en duodeno (correspondió a gema de vidrio que permaneció en duodeno por más de 4 semanas).

El tiempo promedio transcurrido entre la ingesta del cuerpo extraño y la realización del procedimiento endoscópico fue 15 h $54 \mathrm{~min}$ para los CE esofágicos, con un rango que varió entre 2 h 30 min y 48 h (dependiendo de la ubicación y la oportunidad de la consulta, ya que los proximales fueron más precoces). Para los CE gástricos el promedio de espera fue de 124 h (4 h-32 días), correspondiendo principalmente a monedas y 30 días para el CE duodenal referido en el párrafo previo.

Todos los procedimientos se realizaron en sala de procedimientos quirúrgicos, $92 \%$ con anestesia general y el 8\% restante con sedación profunda bajo la supervisión de un anestesista.

Respecto al tipo de CE, 57\% correspondían a monedas principalmente de $\$ 10$ pesos $(21$ $\mathrm{mm})$ y $\$ 100$ pesos $(23-25 \mathrm{~mm})$ y $9 \%$ a pilas de botón, $10 \%$ a objetos plásticos, $6 \%$ a trozos de carne, $4 \%$ a espinas de pescado, y el resto a una bolita de vidrio, un resorte metálico, un aro metálico, un anillo y una medalla (figura 2). En la figura 3 se pueden observar algunos de los cuerpos extraños encontrados en los pacientes de este estudio.

Respecto a la forma de extracción, en la mayoría ( $70 \%$ pacientes) se extrajo con pinza endoscópica extractora de cuerpo extraño (diente ratón o cocodrilo). En 13\% se extrajo con Roth $\mathrm{Net}^{\circledR}$, en $4 \%$ con pinza Magill bajo visión endoscópica directa, en $4 \%$ con asa de polipectomía, y en 7\% se utilizó en conjunto asa y pinza y en 1 paciente se extrajo bajo visión endoscópica con sonda Foley y canastillo de dormía luego de fracasar extracción con instrumentos habituales.

En las lesiones encontradas post endoscopia, se observaron erosiones en $37 \%$ y úlceras en $8 \%$ pacientes. La tabla 1 detalla las lesiones encontradas y en la figura 4 se muestran fotos de 2 pacientes con daño asociado. Ninguno de los pacientes presentó complicaciones derivadas del CE o del procedimiento endoscópico, excepto un niño, que presentó fiebre autolimitada y que cedió en forma espontánea antes de $24 \mathrm{~h}$.

Sólo un paciente presentó 2 CE simultáneos que correspondieron a monedas gástricas.

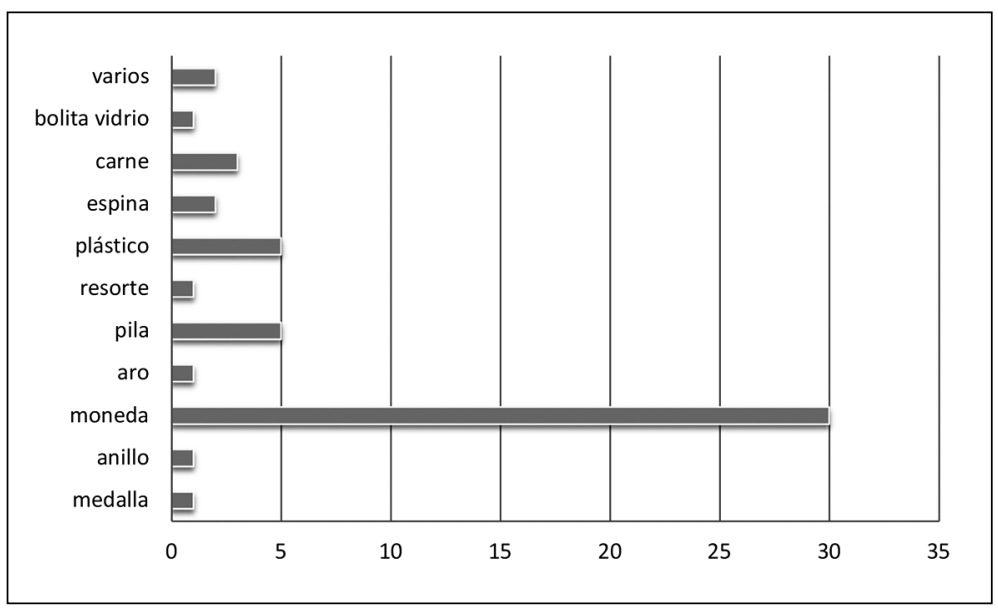

Figura 2. Tipos de cuerpo extraño en 51 pacientes. *Varios: papel, pelusas, pelos, cinta adhesiva. 

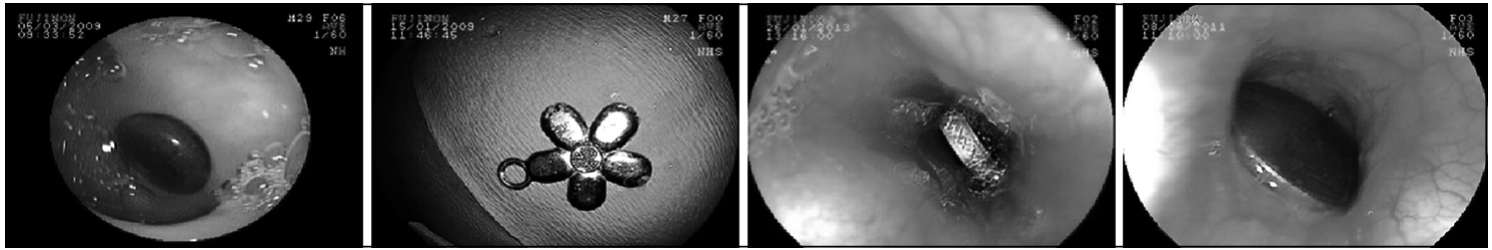

Figura 3. Tipos de cuerpo extraño en 51 pacientes. De izquierda a derecha: bolita de vidrio, medalla, anillo, moneda.
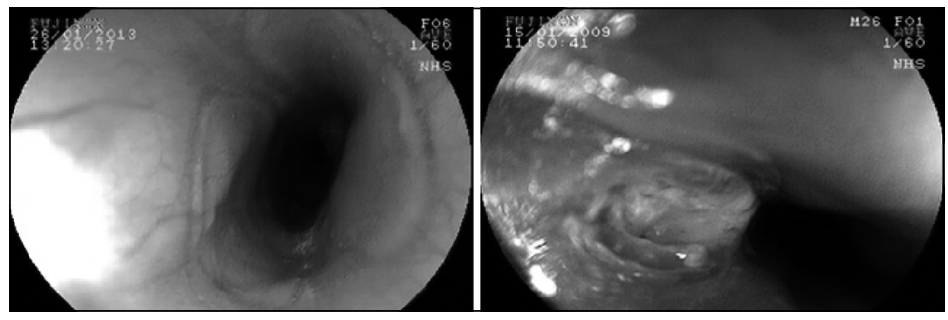

Figura 4. Lesiones encontradas en 51 pacientes con cuerpo extraño digestivo. Izquierda: Erosión esofágica. Derecha: Úlcera esofágica.

Tabla 1. Distribución según tipo de lesión encontrada en control endoscópico posterior a la extracción en 51 pacientes con CE

\begin{tabular}{|c|c|c|}
\hline & $\mathbf{n}$ & $\%$ \\
\hline \\
\hline \multicolumn{3}{|l|}{ Erosiones } \\
\hline Esófago proximal & 13 & 25 \\
\hline medio & 5 & 10 \\
\hline distal & 0 & \\
\hline Estómago & 1 & 2 \\
\hline Duodeno & 0 & \\
\hline \multicolumn{3}{|l|}{ Úlceras } \\
\hline Esófago proximal & 1 & 2 \\
\hline medio & 3 & 6 \\
\hline distal & 0 & \\
\hline Estómago & 0 & \\
\hline Duodeno & 0 & \\
\hline Sin lesiones & 28 & 55 \\
\hline Total & 51 & 100 \\
\hline
\end{tabular}

\section{Discusión}

La literatura describe que aproximadamente $80 \%$ de los CE ingeridos se eliminan espontáneamente y que en un $60 \%$ de ellos, dicha eliminación no es detectada por los padres ${ }^{15,16}$. No contamos con datos estadísticos precisos respecto del número total de consultas por ingesta, o sospecha de ingesta de CE en el período analizado.

Al igual que lo descrito en la literatura la
Tabla 2. Niveles de prioridad para extracción de cuerpo extraño ingeridos*

Prioridad 1: Endoscopia urgente (tiempo espera máximo 4-6 h)

- CE en esófago o estómago con características de riesgo (cortante, punzante) o longitud mayor $5 \mathrm{~cm}$ ( o $3 \mathrm{~cm}$ en lactantes)

Pila/batería botón en esófago

Signos de compromiso aéreo

Signos o síntomas sugerentes de obstrucción esofágica o gastroduodenal

Cualquier CE en esófago tercio superior esofágico

Prioridad 2: Endoscopia no urgente (tiempo de espera máximo 12-24 h)

- CE en esófago medio o distal sin características de riesgo

Comida impactada en esófago sin signos de obstrucción

- CE en estómago de diámetro mayor $2 \mathrm{~cm}$

- Presencia de 2 o más CE imantados en estómago independientes del tamaño

Prioridad 3: Endoscopia diferida (tiempo de espera superior a $48 \mathrm{~h}$ )

Pila/batería botón o cilíndrica en estómago que permanece más de $48 \mathrm{~h}$

Cualquier CE en tracto digestivo accesible endoscópicamente y que persista por más de 4 semanas

*Extraído de referencia 23.

mayor frecuencia de CE digestivo se da en los menores de 3 años dado la tendencia natural de los niños a llevarse las cosas a la boca ${ }^{9,11,17}$.

El riesgo de lesiones está determinado por la ubicación, el tamaño, la forma, la naturaleza 
del CE y el tiempo de contacto con los tejidos. Los objetos de menos de $2 \mathrm{~cm}$ de ancho y hasta $5 \mathrm{~cm}$ de longitud recorren sin impedimentos el tubo digestivo y se eliminan a través de las heces en niños mayores de 2 años, en niños menores de 2 años el límite de longitud observado es de $3 \mathrm{~cm}^{12}$.

En nuestra serie, un pequeño porcentaje de pacientes tenía antecedentes mórbidos de importancia (retraso desarrollo psicomotor y atresia esofágica), descritos como factores de riesgo para la ingesta de $\mathrm{CE}^{3,4}$. Es importante, tener en consideración dichos antecedentes a la hora de evaluar un niño en el servicio de urgencia, debiendo considerarse los CE gastrointestinales como hipótesis diagnóstica, aún cuando no presenten el antecedente de ingesta.

La sintomatología encontrada en nuestros pacientes se corresponde con la literatura ${ }^{5-15}$, aunque siempre hay tener en consideración que un porcentaje importante de niños llega al servicio de urgencia asintomáticos, hasta un $50 \%$ en algunas publicaciones $(15 \%)$. La mayoría de los CE esofágicos se alojan inmediatamente por debajo del músculo cricofaríngeo ocasionado síntomas como náuseas, sialorrea, vómito, hematemesis, dolor abdominal, odinofagia y sensación de opresión traqueal. El segundo sitio más frecuente es la unión esofagogástrica, y los síntomas pueden desaparecer transitoriamente, sin embargo no se debe asumir que el CE ha migrado ${ }^{17}$.

Dado que la mayoría de los CE gastrointestinales son radiopacos ${ }^{15}$, la radiografía simple cervical-torácica y abdominal es muy buen estudio inicial. La proyección lateral es útil porque la super posición de las imágenes puede dificultar la visualización del CE y permite precisar la ubicación de este. En caso se sospechar complicaciones la radiografía también puede detectar presencia de enfisema subcutáneo, neumomediastino y ocasionalmente neumotórax. Es el caso de nuestros pacientes, en $75 \%$ de los CE fueron visibles en la radiografía simple. Cabe destacar eso sí, que una radiografía normal no descarta la presencia de un $\mathrm{CE}$, por lo que otras técnicas diagnósticas pueden ser útiles, como la radiografía con medio de contraste diluido cuya utilidad es discutida por el riesgo de aspiración y dificultad en la visón endoscópica subsecuente, la tomografía computada o incluso la misma EDA, que puede servir como elemento diagnóstico y/o terapéutico ${ }^{7}$. Otro punto a considerar es que no siempre lo que se ve en la radiografía corresponde exactamente al $\mathrm{CE}$ que vamos a encontrar, ya que muchas veces están compuestos de diferentes materiales, algunos de los cuales no son evidenciables mediante radiografía, por lo que el tamaño real puede ser muy superior al sospechado.

La ubicación y los tipos de CE no difieren de las distintas publicaciones, siendo las monedas las más frecuentemente encontradas y localizadas en su mayoría en esófago proxi$\mathrm{mal}^{1,18}$.

Uno de nuestros pacientes, que presentaba un cuerpo extraño duodenal, era portador de un diafragma duodenal no diagnosticado, que se evidenció durante el procedimiento endoscópico de extracción, logrando resolución endoscópica de ambas patologías por la misma vía.

A pesar de que hay variadas técnicas descritas para la extracción de los cuerpos extra$\tilde{n}_{0}{ }^{16}$, la endoscopía es un procedimiento seguro y eficaz ${ }^{19}$, que en nuestra serie no presentó complicaciones posteriores y logró extraer la totalidad de los CE. En 2 casos la EDA apoyo la extracción mediante laringoscopia.

Es ampliamente aceptado que cuando el CE supera las estrecheces naturales del tubo digestivo la conducta debe ser expectante. Por otra parte, en los pacientes que superadas las zonas naturalmente estrechas o aquellos que ingieren cuerpos pequeños en los que no ocurre la migración espontánea, se debe sospechar una causa adquirida o congénita que lo explique (cirugías previas, malformaciones, diafragmas, etc). Otra ventaja que presenta la EDA para el manejo de esta patología, es la posibilidad de poder evaluar directamente la presencia de lesiones y/o complicaciones en la mucosa subyacente al CE.

Las lesiones más frecuentes descritas son pequeñas laceraciones mucosas y peri esofagitis, otras más importantes son la fístula del esófago a la tráquea, el bronquio, a la aorta o incluso al pericardio con taponamiento cardíaco. Pero sin duda la complicación más temida es la perforación esofágica, que puede dar lu- 
gar a mediastinitis y abscesos retro esofágicos, con un alto porcentaje de mortalidad ${ }^{6,20-22}$. Las perforaciones se pueden producir bien por acción del cuerpo extraño: i) por sus características morfológicas (objeto cortante o punzante) y ii) por erosión progresiva o acción química del CE o sus componentes al permanecer mucho tiempo en contacto con la mucosa esofágica. La lesión esofágica también puede ser de origen iatrogénico durante el procedimiento de extracción.

En general recomendamos fuertemente realizar el procedimiento endoscópico con anestesia general e intubación oro traqueal. En caso los raros casos que la extracción endoscópica sea urgente (ej. batería de botón impactada) puede estar indicado realizar EDA sin completar ayuno. Solo excepcionalmente realizamos la extracción bajo sedación profunda y esto dependerá de las condiciones del paciente y de la experiencia tanto del anestesista como del endoscopista, preocupándose de que se realice bajo estrictos estándares de seguridad para el paciente y la mayor comodidad para los intervencionistas de modo de asegurar el mejor resultado y prevenir la aparición de complicaciones.

El momento o la oportunidad del procedimiento están determinados por la localización, síntomas y tipo de CE. En general los pacientes sintomáticos, la presencia de pilas y objetos cortantes en esófago requieren procedimiento de urgencia. En la tabla 2 se describen tiempos para realizar EDA según sociedad española de Gastroenterología Infantil ${ }^{23}$. Las recomendaciones de manejo según las guías publicadas, varían también en los distintos artículos, pero en términos generales, se recomienda que los CE esofágicos, se extraigan antes de las $24 \mathrm{~h}$ de ingeridos. Los resultados encontrados en nuestra serie se adecuan a las guías internacionales, representando la buena disponibilidad del equipo de trabajo y equipamiento para la atención de nuestros niños en un servicio público.

La seguridad del procedimiento se ha mejorado con mejor equipamiento y la formación de equipos. La utilización del sobre tubo puede ser muy útil para la extracción de cuerpos punzantes, pero no siempre puede utilizarse en niños pequeños. En nuestros pacientes no se utilizo pese a contar con el equipamiento. Entre los accesorios disponibles existen pinzas, canatos, asas, cuya utilidad dependerá del tipo de CE y la preferencia del endoscopista.

Una vez realizada la extracción siempre se debe evaluar el estado de la mucosa por posibles complicaciones y detectar presencia de lesiones previas congénitas o adquiridas. A diferencia de otros reportes nacionales ${ }^{11}$, la presencia de lesiones post procedimiento en esta serie sólo fue de $43 \%$.

En especial debe considerarse 2 situaciones: la ingesta de pilas/baterías e imanes. La ingesta de pilas/baterías puede causar daño de la mucosa asociado a lesiones por decúbito o quemaduras de bajo voltaje. Respecto a los imanes su riesgo reside en la ingesta de 2 o más cuerpos imantados por separado o acompañados de otros objetos metálicos dada la posibilidad de que se produzcan perforaciones en el intestino al atraerse entre ellos con interposición de pared intestinal con posterior perforación $^{6,12,21,22,24}$.

En conclusión, la prevención es la estrategia fundamental para disminuir la morbimortalidad secundaria a la ingestión de $\mathrm{CE}$ en la infancia. Para conseguir lo anterior, no sólo deben generarse políticas públicas y reglamentos que regulen tipos de juguetes según edades, sino fundamentalmente educar a la población en forma eficiente, informando de los riesgos y consecuencias que puede tener este accidente potencialmente evitable. En caso de tener a un paciente con sospecha o confirmación de CE, la ubicación, tipo de CE, tiempo transcurrido y disponibilidad de recursos, deben orientar la conducta más apropiada.

Potenciales conflictos de interés: Este trabajo cumple con los requisitos sobre consentimiento/asentimiento informado, comité de ética, financiamiento, estudios animales y sobre la ausencia de conflictos de intereses según corresponda.

Financiamiento: sin financiamiento.

\section{Referencias}

1.- Chung S, et al: A Review of Pediatric Foreign Body Ingestion and Management. Clin Pediatr Emerg Med 2010; 11:225-30. 
2.- Shenghong Z, Yi C, Xiao Gong G, Fang G, Minhu C, Bihui Z: Endoscopic Management of Foreign Bodies in the Upper Gastrointestinal Tract in South China: A Retrospective Study of 561 Cases. Dig Dis Sci 2010; 55: 1305-12.

3.- Alliendes $F$, et al: Ingestión de cuerpos extraños. Rev Chil Pediatr 1999; 70 (4): 335-6.

4.- Llompart A, et al: Abordaje endoscópico de los cuerpos extraños esofágicos. Resultados de una serie retrospectiva de 501 casos. Gastroenterol Hepatol 2002; 25 (7): 448-51.

5.- Asraf O: Foreign body in the esophagus: a review. Sao Paulo Med J 2006; 124 (6): 346-9.

6.- Kay M, Wyllie R: Pediatric Foreign Bodies and their management. Current Gastroenterology Reports 2005; 7: 212-8.

7.- Ikenberry S, Jue T, Andersen M, et al: Management of ingested foreign bodies and food impactions. Gastrointestinal endoscopy 2011; 73 (6): 1085-91.

8.- McNeill MB, et al: Epidemiology and management of esophageal coin impaction in children. Digestive and Liver Disease 2012; 44: 482-6.

9.- Ríos C, Correia $G$ : Cuerpos extraños en pacientes pediátricos 5 años de revisión. Rev Ped Elec [En línea] 2008; 5 (3): 25-31.

10.- Shivakumar M, Naik A, Prashanth K, Yogesh B, Hongal $G$ : Foreign Body in Upper Digestive Tract. Indian J Pediatr 2004; 71 (8): 689-93.

11.- Ríos G, Alliende F, Miquel I, Arancibia ME, Rodríguez L, Saelzer E: Extracción Endoscópica De Cuerpos Extraños Digestivos. Rev Chil Pediatr 2013; 84 (5): 505-12.

12.- Litovitz TL, Klei-Schwartz W, White S, et al: 2000 annual report of the American Association of Poison Control Center Toxic Exposure Surveillance System. Am J Emerg Med 2001; 19 (5): 337-95.
13.- Yalcin $S$, et al: Foreign body ingestion in children: an analysis of pediatric surgical practice. Pediatr Surg Int 2007; 23: 755-61.

14.- Panieri E, et al: The management of ingested bodies in children. A review of 663 cases. Eur J Emerg Med 1995; 2: 83-7.

15.- Maluenda $C$, et al: Ingesta de cuerpos extraños. Protocolos de Gastroenterología, Hepatología y Nutrición Pediátrica 2010. $2^{\circ}$ Edicion; Capítulo 15: 131-4.

16.- Chung $S$, et al: A Review of Pediatric Foreign Body Ingestion and Management. Clinical Pediatric Emergency Medicine 2010; 11 (3): 225-30.

17.- Alvarado $U$, et al: Cuerpos extraños alojados en las vías aérea y digestiva. Diagnóstico y tratamiento. Acta Pediatr Mex 2011; 32 (2): 93-100.

18.- Marisa $C$, et al: Foreign Body Ingestion and Aspiration. Pediatr Rev 2009; 30: 295-301.

19.- Uyemura MC: Foreign Body Ingestion in Children. Am Fam Physician 2005; 72: 287-91.

20.- Paulino A, Fernández J, Arroyo B, Sánchez F, De Saa $M$, García R: Cuerpos extraños esofágicos. Medicina General 2002; 47: 660-3.

21.- Cortés $C$, Silva $C$ : Ingestión accidental de imanes en niños y sus complicaciones: Un riesgo creciente. Rev Med Chile 2006; 134: 1315-9.

22.- Little DC, et al: Esophageal foreign bodies in the pediatric population: our first 500 cases. J Pediatr Surg 2006; 41: 914-8.

23.- Villa V, Bodas A, Rodríguez A: Ingesta de Cuerpos extraños. En Sociedad Española de gastroenterología, Hepatología y Nutrición: Tratamiento en Gastroenterología Hepatología y Nutrición Pediátrica. Madrid. Editorial ERGON, $3^{\circ}$ edición 2012; 342-50.

24.- Goh B, et al: Perforation of the Gastrointestinal Tract Secondary to Ingestion of Foreign Bodies. World J Surg 2006; 30: 372-7. 